TOHO-FP-9861

YITP-98-33

\title{
Electric-Magnetic Duality Rotations and Invariance of Actions
}

\author{
Yuji Igarashi, Katsumi Itoh ${ }^{a}$ and Kiyoshi Kamimura ${ }^{b}$ \\ Faculty of Education, Niigata University, Niigata 950-21, Japan \\ ${ }^{a}$ Yukawa Institute for Theoretical Physics, Kyoto University \\ Kyoto 606-01, Japan \\ ${ }^{b}$ Department of Physics, Toho University, Funabashi 274, Japan
}

\begin{abstract}
For $\mathrm{D}=4$ theories of a single $\mathrm{U}(1)$ gauge field strength coupled to gravity and matters, we show that the electric-magnetic duality can be formulated as an invariance of the actions. The symmetry is associated with duality rotation acting directly on the gauge field. The rotation is constructed in flat space, and an extension to curved spaces is also given. It is non-local and non-covariant, yet generates off-shell extended transformation of the field strength. The algebraic condition of Gaillard and Zumino turns out to be a necessary and sufficient condition for the invariance of actions. It may be used as a guiding principle in constructing self-dual actions in string and field theories.
\end{abstract}

PACS: 11.15.-q; 11.10.Ef

Keywords: Duality in Gauge Field Theories; D-branes; String Duality

\footnotetext{
${ }^{1}$ Permanent address: Faculty of Education, Niigata University, Niigata 950-21, Japan
} 


\section{Introduction}

Duality symmetries which relate strong couplings to weak couplings have been considered as a key concept in understanding non-perturbative aspects of string and field theories. The $\mathrm{D}=4$ electric-magnetic duality is the prototype of the symmetries. It is associated with a rotation between electric and magnetic fields, described by an infinitesimal transformation of field strength to be called as the F-transformation in this paper. In the presence of matter fields, the rotation should be accompanied, in general, by their appropriate transformations. The duality is not known to be manifest in the action but only as a symmetry of equations of motion (EOM). In view of increasing recognition of its importance, it is certainly desirable to incorporate this symmetry directly into the action.

The purpose of this paper is to show that the duality can be formulated as a pseudoinvariance' of a generic action for interacting U(1) gauge field strength with gravity and matters. There are two crucial issues in this formulation. The first is to realize the importance of the Gaillard-Zumino (GZ) condition[1] [2] on the action.2] It was originally found as a condition for the invariance of the stationary surface. Obviously it is the necessary condition for the pseudo-invariance of the action. We show that the GZ condition is at the same time a sufficient condition: if the action satisfies the GZ condition, it becomes pseudo-invariant. One needs to specify the transformations of matter fields so as to be consistent with the condition. Therefore, this algebraic relation provides us with a criterion in construction of self-dual actions in string and field theories. The second is to consider a duality transformation of the fundamental dynamical variable, the gauge field, rather than that of the field strength. It is a natural extension of the one given by Deser and Teitelboim [4], referred to as A-transformation in this paper, being non-local and non-covariant, yet allows us to formulate the duality as an invariance of the action. The A-transformation is first constructed in flat space, and extended to generic curved spaces under some assumptions on the inverse Laplacian operator for $\mathrm{D}=3$ spatial vectors. Our formulation given here should be compared with models with dual gauge fields such as the Schwarz-Sen model[5], and its covariant version of PST [6]. Such approaches of doubling gauge fields may be used to avoid above mentioned non-locality and the sacrifice of $\mathrm{D}=4$ manifest covariance. It seems however that general criterion for constructing self-dual actions with matter fields such as the GZ condition is not known in the approaches with dual gauge fields.

We stress that since the F-transformation mixes the Bianchi identities with the EOM, it is only sensible for the on-shell gauge field configurations. Unlike this, the A-transformation is constructed so as to admit off-shell field configurations, and to induce the F-transformation on the mass shell. The action becomes pseudo-invariant under the A-transformation, if it obeys the GZ condition. As a non-trivial application of the formulation given here, we show in a subsequent publication[7] that the super D3-brane action satisfies the GZ condition, and therefore is exactly self-dual. A new feature found in that work is that

\footnotetext{
${ }^{1}$ When an action is not exactly invariant but transforms by a surface term, it is said to be pseudoinvariant.

${ }^{2}$ This condition was discussed also by Gibbons and Rasheed[3] in the absence of matter fields.
} 
fermionic co-ordinate of the brane should transform while their bosonic super partner are left invariant under the duality transformation. This proof of self-duality can be done without resort to any semi-classical approximations in contrast to earlier works.

In our formulation based on the A-transformation, pseudo-invariance of the action in the Lagrangian formalism implies an invariance of the Hamiltonian in the canonical formalism. It has been discussed that the Maxwell action is not pseudo-invariant under a finite duality transformation, while the Hamiltonian is invariant. The source of this conundrum is the use of a finite F-transformation. In the A-transformation, there is no such discrepancy between the Lagrangian and Hamiltonian approaches. In the latter, the A-transformation is shown to be described as a canonical transformation. Therefore, the criterion for self-duality in the canonical formalism is commutability of the Hamiltonian with the generator of the canonical transformation.

This paper is organized as follows. The next section describes the F-transformation and the GZ condition. In section 3, A-transformation in the flat space-time is given. We discuss a curved space-time extension of the A-transformation in section 4 . We also show that the A-transformation is described as a canonical transformation. Summary and discussion is given in section 5. In Appendix A, we show that the GZ condition is an algebraic relation which is not restricted to the on-shell fields. Some formulae used in the $\mathrm{D}=3$ covariant calculations are summarized in Appendices B and C.

\section{F-transformation and the Gaillard-Zumino Condi- tion}

We begin with a brief review of duality transformation on field strength and the GZ condition. It was shown [1] [2] that the maximal duality group associated with infinitesimal transformations of $n \mathrm{U}(1)$ field strengths is $\operatorname{Sp}(2 \mathrm{n}, \mathrm{R})$. This non-compact group is realized only when there are appropriate scalar fields in the theory. In the absence of them, the relevant group is its maximal compact subgroup, U(n). Conversely, the U(n) duality can be lifted to the non-compact $\operatorname{Sp}(2 \mathrm{n}, \mathrm{R})$ duality by including some scalar fields, as discussed by Gibbons and Rasheed[8] and Gaillard and Zumino[2]. We consider here for simplicity an $\mathrm{SO}(2)$ duality for a single gauge field strength $F_{\mu \nu}$ coupled with $\mathrm{D}=4$ metric $g_{\mu \nu}$ and matter fields $\Phi^{A}$. Given a generic Lagrangian density $\mathcal{L}\left(F_{\mu \nu}, g_{\mu \nu}, \Phi^{A}\right)=$ $\sqrt{-g} L\left(F_{\mu \nu}, g_{\mu \nu}, \Phi^{A}\right)$, one may define the constitutive relation by

$$
\tilde{K}^{\mu \nu}=\frac{\partial L}{\partial F_{\mu \nu}}, \quad \frac{\partial F_{\alpha \beta}}{\partial F_{\mu \nu}}=\left(\delta_{\alpha}^{\mu} \delta_{\beta}^{\nu}-\delta_{\beta}^{\mu} \delta_{\alpha}^{\nu}\right),
$$


where the Hodge dual components for the anti-symmetric tensor $K_{\mu \nu}$ are given by

$$
\tilde{K}_{\mu \nu}=\frac{1}{2} \eta_{\mu \nu}^{\rho \sigma} K_{\rho \sigma}, \quad K_{\mu \nu}=-\frac{1}{2} \eta_{\mu \nu}^{\rho \sigma} \tilde{K}_{\rho \sigma}, \quad \tilde{\tilde{K}}_{\mu \nu}=-K_{\mu \nu}
$$

In terms of $F_{\mu \nu}$ and $K_{\mu \nu}$, the EOM for gauge field and the Bianchi identity read

$$
\begin{aligned}
& \partial_{\mu}\left(\sqrt{-g} \tilde{K}^{\mu \nu}\right)=\frac{1}{2} \epsilon^{\mu \nu \rho \sigma} \partial_{\mu} K_{\rho \sigma}=0 \\
& \partial_{\mu}\left(\sqrt{-g} \tilde{F}^{\mu \nu}\right)=\frac{1}{2} \epsilon^{\mu \nu \rho \sigma} \partial_{\mu} F_{\rho \sigma}=0 .
\end{aligned}
$$

The $\mathrm{SO}(2)$ duality has been postulated as a symmetry of the EOM: its infinitesimal form is

$$
\delta F=\lambda K, \quad \delta K=-\lambda F
$$

associated with

$$
\delta \Phi^{A}=\xi^{A}(\Phi), \quad \delta g_{\mu \nu}=0
$$

where $\xi^{A}(\Phi)$ should depend neither derivatives of the fields, $\partial_{\mu} \Phi^{A}=\Phi_{\mu}^{A}$, nor other fields, $g_{\mu \nu}$ and $F_{\mu \nu}$. Note that $F_{\mu \nu}$ and $K_{\mu \nu}$ are not independent one another but non-linearly related in general. Therefore, under (2.4), only a restricted class of actions will make the relation (2.1) invariant. This constraint on actions is the GZ condition, which may be obtained out of some equations given in refs.[1]]2]. For $\mathrm{SO}(2)$ duality, it is given by

$$
\frac{\lambda}{4}(F \tilde{F}+K \tilde{K})+\delta_{\Phi} L=0
$$

where $F \tilde{F}=F_{\mu \nu} \tilde{F}^{\mu \nu}$.

The GZ condition can be derived by requiring:

(1) invariance of the constitutive relation (2.1);

(2) covariance for the EOM of matter fields [1] [2],

$$
\delta[\mathcal{L}]_{A}=-\frac{\partial \xi^{B}}{\partial \Phi^{A}}[\mathcal{L}]_{B}, \quad[\mathcal{L}]_{A}=\frac{\partial \mathcal{L}}{\partial \Phi^{A}}-\partial_{\mu} \frac{\partial \mathcal{L}}{\partial \Phi_{\mu}^{A}}
$$

(3) invariance of the EOM for the metric $g_{\mu \nu}$, which implies invariance of the energymomentum tensor

$$
\delta T^{\mu \nu}=0, \quad \frac{\delta S}{\delta g_{\mu \nu}}=-\frac{\sqrt{-g}}{2} T^{\mu \nu}
$$

under the duality transformation (2.4) and (2.5).

\footnotetext{
${ }^{3}$ Here we use the following convention: $\eta^{\mu \nu \rho \sigma}$ denotes the covariantly constant anti-symmetric tensor with indices raised and lowered using the metric $g_{\mu \nu}$ whose signature is $(-+++)$. We also use the tensor densities $\epsilon^{\mu \nu \rho \sigma}$ and $\epsilon_{\mu \nu \rho \sigma}$ with weight -1 and 1 . In terms of $g=\operatorname{det} g_{\mu \nu}$, they are defined by $\epsilon^{\mu \nu \rho \sigma}=\sqrt{-g} \eta^{\mu \nu \rho \sigma}$ and $\eta_{\mu \nu \rho \sigma}=\sqrt{-g} \epsilon_{\mu \nu \rho \sigma}$, normalized as $\epsilon^{0123}=-\epsilon_{0123}=1$.
} 
First, one finds the constitutive relation (2.1) to transform as

$$
\delta \tilde{K}=\frac{1}{2} \frac{\partial \tilde{K}}{\partial F} \lambda K+\frac{\partial}{\partial F} \delta_{\Phi} L=-\lambda \tilde{F}+\frac{\partial}{\partial F} \beta,
$$

where

$$
\beta \equiv \frac{\lambda}{4}(\tilde{K} K+\tilde{F} F)+\delta_{\Phi} L
$$

As shown in Appendix A, the EOMs for the matter fields change as

$$
\delta[\mathcal{L}]_{A}=-\frac{\partial \xi^{B}}{\partial \Phi^{A}}[\mathcal{L}]_{A}+[\sqrt{-g} \beta]_{A} .
$$

We also find

$$
\begin{aligned}
\delta\left(\frac{\delta S}{\delta g_{\mu \nu}(x)}\right) & =\frac{1}{2} \lambda \int d^{4} y\left(K_{\rho \sigma}(y) \frac{\delta}{\delta g_{\mu \nu}(x)}\left(\sqrt{-} g \tilde{K}^{\rho \sigma}\right)(y)+\frac{\delta}{\delta g_{\mu \nu}(x)} \delta_{\Phi} \mathcal{L}(y)\right) \\
& =\frac{\delta}{\delta g_{\mu \nu}(x)} \int d^{4} y(\sqrt{-g} \beta)(y) .
\end{aligned}
$$

The above three conditions 1) $\sim 3$ ) require that $\sqrt{-g} \beta$ should be a constant. Moreover, in order for the relation (2.10) to have a consistent power series expansion in terms of $\left(F, \Phi^{A}, \Phi_{\mu}^{A}\right)$, the constant should be zero, so that $\beta=0$. This leads to the GZ condition.

As mentioned in the previous section, the F-transformation is only sensible for onshell gauge field configurations. So one might think, from the above derivation, that the GZ condition holds only for on-shell fields. However, it is not so: the GZ condition is an algebraic relation that is free from on-shell conditions in spite of its derivation. In Appendix A, we show it explicitly in connection with A-transformation which generates off-shell extended transformations of the field strength.

Consider the on-shell gauge field configurations appeared in the F-transformation, and suppose that the matter fields are absent. Then the Lagrangian density transforms by a term proportional to $\sqrt{-g} K \tilde{K}$. In the absence of matter fields, the change of the Lagrangian density under the F-transformation is $\sqrt{-g} K \tilde{K}$ or $K_{(2)}^{2}$ in terms of the differential two form $K_{(2)}$. Since the transformation is sensible only for the on-shell gauge configuration, i.e., $d K_{(2)}=0, K_{(2)}^{2}$ becomes exact if the cohomology of $K_{(2)}^{2}$ is trivial. In this sence, the pseudo-invariance of the action under the F-transformation trivially follows. What is non-trivial is that even in the presence of the (off-shell) matter fields, the action still remains pseudo-invariant, if it obeys the GZ condition. This is why Gaillard and Zumino [1] [2] were able to construct a conserved charge associated with the F-transformation. In any case, however, the restriction on the on-shell variation for the gauge field in the F-transformation would be undesirable. This tempts us into considering the A-transformation. We consider it below first in flat space and then in curved spaces. 


\section{A-transformation in flat space}

In order to see the essential issue of duality rotation on the gauge field as simple as possible, and to avoid technical complications appeared in curved spaces, we first consider the A-transformation in flat space[四]. We construct it in such a way that:

1) it allows to include off-shell field configurations and produces the F-transformation on the mass-shell;

2) a generic action becomes pseudo-invariant if the GZ condition is satisfied.

For $\mathrm{SO}(2)$ duality, the A-transformation which satisfies the above requirements is given in a non-covariant $(3+1)$ decomposition by

$$
\begin{aligned}
\delta A_{i} & =-\lambda \epsilon_{i j k} \partial^{-2} \partial^{j} \tilde{K}^{0 k}, \quad(i, j, k=1,2,3) \\
\delta A_{0} & =-\frac{\lambda}{2} \partial^{-2} \partial^{k}\left(\epsilon_{i j k} \tilde{K}^{i j}\right),
\end{aligned}
$$

where $\tilde{K}^{\mu \nu}$ is defined by (2.1) in flat space and is in general a non-linear function of $\left(F, \Phi^{A}, \partial \Phi^{A}\right)$. In relation to the inverse Laplacian operator $\partial^{-2}$ we assume that: a) non-trivial kernel does not exist; b) it commutes with derivatives, $\partial_{\mu} \partial^{-2}=\partial^{-2} \partial_{\mu}$, and allows the partial integration, $f \partial^{-2} g=\left(\partial^{-2} f\right) g+$ (surface term).

The A-transformation (3.1) generates changes in the field strength

$$
\begin{aligned}
\delta F_{i j} & =\lambda \epsilon_{i j k} \tilde{K}_{\perp}^{0 k} \\
& =\lambda K_{i j}+\lambda \epsilon_{i j k} \partial^{-2} \partial^{k}\left[\partial_{\mu} \tilde{K}^{\mu 0}\right], \\
\delta F_{0 i} & =\partial_{0}\left(-\lambda \epsilon_{i j k} \partial^{-2} \partial^{j} \tilde{K}^{0 k}\right)-\partial_{i}\left(-\frac{\lambda}{2} \partial^{-2} \partial^{j}\left(\epsilon_{j k \ell} \tilde{K}^{k \ell}\right)\right) \\
& =\lambda K_{0 i}-\lambda \epsilon_{i j k} \partial^{-2} \partial^{j}\left[\partial_{\mu} \tilde{K}^{\mu k}\right],
\end{aligned}
$$

where [ ] indicates contributions which vanish on the mass-shell. The use of EOM reduces (3.2) to $\delta F_{\mu \nu}=\lambda K_{\mu \nu}$. As shown in Appendix A, the GZ condition (2.6) and (3.2) lead to

$$
\delta \tilde{K}^{\mu \nu}=-\lambda \tilde{F}^{\mu \nu}+\frac{1}{2} \frac{\partial \tilde{K}^{\rho \sigma}}{\partial F_{\mu \nu}}[E O M]_{\rho \sigma},
$$

where $[E O M]_{\rho \sigma}$ are the components of EOM in (3.2). This gives rise to $\delta \tilde{K}^{\mu \nu}=-\lambda \tilde{F}^{\mu \nu}$ on the mass shell again. One finds that the A-transformation (3.1) satisfies the requirement 1).

We next consider the change of the Lagrangian under (3.1):

$$
\begin{aligned}
\delta L= & \frac{1}{2} \frac{\partial L}{\partial F_{\mu \nu}} \delta F_{\mu \nu}+\delta_{\Phi} L \\
= & \tilde{K}^{0 i}\left(\lambda K_{0 i}-\lambda \epsilon_{i j k} \partial^{-2} \partial^{j}\left[\partial_{\mu} \tilde{K}^{\mu k}\right]\right) \\
& +\frac{1}{2} \tilde{K}^{i j}\left(\lambda K_{i j}+\lambda \epsilon_{i j k} \partial^{-2} \partial^{k}\left[\partial_{\mu} \tilde{K}^{\mu 0}\right]\right)+\delta_{\Phi} L
\end{aligned}
$$




$$
\begin{aligned}
= & \frac{\lambda}{2} \tilde{K} K+\delta_{\Phi} L-\lambda \tilde{K}^{0 i} \epsilon_{i j k} \partial^{-2} \partial^{j}\left[\partial_{\mu} \tilde{K}^{\mu k}\right] \\
& +\frac{1}{2} \lambda \tilde{K}^{i j} \epsilon_{i j k} \partial^{-2} \partial^{k}\left[\partial_{\mu} \tilde{K}^{\mu 0}\right] .
\end{aligned}
$$

The last two terms proportional to the EOM become

$$
-\frac{\lambda}{4} \tilde{K} K+\partial_{0}\left(-\frac{\lambda}{2} \tilde{K}^{0 i} \epsilon_{i j k} \partial^{j} \partial^{-2} \tilde{K}^{0 k}\right)
$$

up to a total spatial derivative. Imposing the GZ condition (2.6), the Lagrangian is shown to transform by a total $\mathrm{D}=4$ divergence:

$$
\begin{aligned}
\delta L & =\partial_{0}\left(-\frac{\lambda}{2} \tilde{K}^{0 i} \epsilon_{i j k} \partial^{-2} \partial^{j} \tilde{K}^{0 k}-\frac{\lambda}{2} A_{i} \epsilon^{i j k} \partial_{j} A_{k}\right)+\partial_{i} U^{i} \\
& \equiv \partial_{\mu} U^{\mu}
\end{aligned}
$$

for some $U^{i}$. One confirms that the action becomes pseudo-invariant for off-shell gauge field under the A-transformation, if it obeys the GZ condition.

The pseudo-invariance of the action leads to the conserved current, $\partial_{\mu} j^{\mu}=0$. In particular, the charge density is given by

$$
\begin{aligned}
j^{0} & =\tilde{K}^{0 i} \delta A_{i}+\frac{\partial L}{\partial \dot{\Phi}^{A}} \delta \Phi^{A}-U^{0} \\
& =-\frac{\lambda}{2} \tilde{K}^{0 i} \epsilon_{i j k} \partial^{-2} \partial^{j} \tilde{K}^{0 k}+\frac{\lambda}{2} A_{i} \epsilon^{i j k} \partial_{j} A_{k}+\frac{\partial L}{\partial \dot{\Phi}^{A}} \delta \Phi^{A} .
\end{aligned}
$$

The discussion given here in the flat space-time shows that the duality symmetry can be formulated as a pseudo-invariance of the action if the GZ condition is fulfilled. Therefore the GZ condition, though originally derived as a necessary condition, is a sufficient condition for the pseudo-invariance for the action as well. It is recognized to be a criterion for the pseudo-invariance of the action. We now consider an extension of the formulation in curved spaces.

\section{Extension to curved space}

In this section, we construct a curved-space version of the A-transformation, where covariance with respect to $\mathrm{D}=3$ subspace is maintained. Basic notations and formulae needed to perform $\mathrm{D}=3$ covariant computations are summarized in Appendix B.

One of the basic operators in our formalism is a curved space extension of the Laplacian operator, $(\tilde{\Delta})_{j}{ }^{i}$, which maps a vector $T_{i}$ into a vector $(\tilde{\Delta}){ }_{j}{ }^{i} T_{i}$. It is given by

$$
(\tilde{\Delta})_{j}{ }^{i}=\Delta \delta_{j}{ }^{i}-R_{j}{ }^{i}, \quad \Delta=\nabla^{j} \nabla_{j} .
$$


where $R_{j}{ }^{i}$ is the Ricci tensor. We assume that boundary conditions can be arranged so that the Laplacian operator has no non-trivial kernel, and its inverse, $\left(\tilde{\Delta}^{-1}\right)_{i}{ }^{j}$, is welldefined.

In terms of this inverse operator, the transformation for spatial components of the gauge field is given by

$$
\delta A_{\ell}=\lambda\left(\tilde{\Delta}^{-1}\right)_{\ell}{ }^{k} \nabla^{j} \epsilon_{j k m} \tilde{\mathcal{K}}^{0 m}=D_{\ell m}^{-1}\left(\lambda \frac{\tilde{\mathcal{K}}^{0 m}}{\sqrt{\gamma}}\right) \equiv \lambda Z_{l},
$$

where $\tilde{\mathcal{K}}^{0 i}$ is a $\mathrm{D}=3$ vector density defined via $\mathrm{D}=4$ tensor density

$$
\tilde{\mathcal{K}}^{\mu \nu}=\sqrt{-g} \tilde{K}^{\mu \nu}
$$

Note that $\tilde{\mathcal{K}}^{0 i}$ divided by $\sqrt{\gamma}=\sqrt{\operatorname{det} g_{i j}}$ becomes a $\mathrm{D}=3$ vector. In (4.2), $D_{i \ell}^{-1}$ is a tensor operator acting on a vector,

$$
D_{i \ell}^{-1} \equiv\left(\tilde{\Delta}^{-1}\right)_{i}{ }^{k} \nabla^{j} \eta_{j k \ell}=\eta_{i j k} \nabla^{k}\left(\tilde{\Delta}^{-1}\right)_{\ell}^{j},
$$

where $\eta_{j k \ell}=\epsilon_{j k \ell} \sqrt{\gamma}$ is the covariantly constant anti-symmetric tensor. $D_{i \ell}^{-1}$ is the inverse of

$$
D^{j k}=\eta^{j \ell k} \nabla_{\ell}=\nabla_{\ell} \eta^{j \ell k}
$$

in a projected space,

$$
\begin{aligned}
D_{i m}^{-1} D^{m k} & =O_{i}{ }^{k}(\nabla), \quad D^{i m} D_{m k}^{-1}=O^{i}{ }_{k}(\nabla), \\
O_{i}{ }^{k}(\nabla) & =\delta_{i}{ }^{k}-\nabla_{i}\left(\Delta^{-1}\right) \nabla^{k} .
\end{aligned}
$$

Note that the operator $O_{i}{ }^{k}(\nabla)$ projects out any longitudinal component defined with the covariant derivative $\nabla_{i}$. These tensor operators satisfy the transverse condition

$$
\nabla^{k} D_{k \ell}^{-1} T^{\ell}=\nabla_{k} D^{k \ell} T_{\ell}=0
$$

Computing $\nabla^{i} \delta A_{i}$ and $D^{i j} \delta A_{j}$, one obtains the transverse condition

$$
\nabla^{i} \delta A_{i}=0
$$

and the transformation of the magnetic field

$$
\delta F_{i j}=\lambda \epsilon_{i j k} \tilde{\mathcal{K}}_{\perp}^{0 k}
$$

where

$$
\begin{aligned}
\tilde{\mathcal{K}}_{\perp}^{0 k} & =\tilde{\mathcal{K}}^{0 k}-\sqrt{\gamma} \nabla^{k}\left(\Delta^{-1}\right)\left(\nabla_{m} \frac{\mathcal{K}^{0 m}}{\sqrt{\gamma}}\right) \\
& =\epsilon^{k \ell m} \partial_{\ell} Z_{m} .
\end{aligned}
$$


The transformation of the time component is given by

$$
\delta A_{0}=-\frac{\lambda}{2}\left[\nabla^{\ell}\left(\tilde{\Delta}^{-1}\right)_{\ell}^{k}\left(\epsilon_{i j k} \tilde{\mathcal{K}}^{i j}\right)\right]+\lambda\left[\left(\Delta^{-1}\right) \nabla^{k}\left(\partial_{0} Z_{k}\right)\right]
$$

It leads to

$$
\begin{aligned}
\delta F_{0 i} & =\partial_{0} \delta A_{i}-\partial_{i} \delta A_{0} \\
& =\lambda K_{0 i}+\lambda D_{i j}^{-1}\left(\frac{\partial_{\mu} \tilde{\mathcal{K}}^{\mu j}}{\sqrt{\gamma}}\right)+\lambda O_{i}{ }^{k} \partial_{0}\left(\frac{D_{k j}^{-1}}{\sqrt{\gamma}}\right) \tilde{\mathcal{K}}^{0 j}
\end{aligned}
$$

where $O^{i}{ }_{k}$ is the transverse projection in (4.6). As shown in Appendix C, the last two terms vanish on the mass shell, leaving the desired first term.

We now consider the variation of the Lagrangian density given by

$$
\begin{aligned}
\delta \mathcal{L} & =\tilde{\mathcal{K}}^{0 i} \delta F_{0 i}+\frac{1}{2} \tilde{\mathcal{K}}^{i j} \delta F_{i j}+\delta_{\Phi} \mathcal{L} \\
& =\tilde{\mathcal{K}}^{0 i} \partial_{0} \delta A_{i}-\tilde{\mathcal{K}}^{0 i} \partial_{i} \delta A_{0}+\frac{1}{2} \tilde{\mathcal{K}}^{i j}\left(\lambda \epsilon_{i j k} \tilde{\mathcal{K}}_{\perp}^{0 k}\right)+\delta_{\Phi} \mathcal{L}
\end{aligned}
$$

One introduces here an equality up to a spatial total derivative, $\sim: A \sim B \Leftrightarrow A=B+\partial_{i} V^{i}$ for some $V^{i}$. Then, one rewrites the first two terms

$$
\begin{aligned}
\tilde{\mathcal{K}}^{0 i} \partial_{t} \delta A_{i}-\tilde{\mathcal{K}}^{0 i} \partial_{i} \delta A_{0} \sim & \partial_{0}\left[\frac{\lambda}{2} Z_{i} \epsilon^{i j k} \partial_{j} Z_{k}\right] \\
+ & \left(\partial_{i} \tilde{\mathcal{K}}^{0 i}\right)\left[\delta A_{0}-\left(\Delta^{-1}\right) \nabla^{k}\left(\partial_{0} \delta A_{k}\right)\right]
\end{aligned}
$$

and the third term

$$
\begin{aligned}
\frac{1}{2} \tilde{\mathcal{K}}^{i j} \delta F_{i j} & =\frac{\lambda}{2} \tilde{\mathcal{K}}^{i j} \epsilon_{i j k} \tilde{\mathcal{K}}_{\perp}^{0 k} \\
& =\frac{\lambda}{2} \tilde{\mathcal{K}}^{i j} \epsilon_{i j k} \tilde{\mathcal{K}}^{0 k}-\frac{\lambda}{2} \tilde{\mathcal{K}}^{i j} \epsilon_{i j k} \sqrt{\gamma}\left(\tilde{\Delta}^{-1}\right)^{k}{ }_{\ell} \nabla^{\ell}\left(\nabla_{m} \frac{\tilde{\mathcal{K}}^{0 m}}{\sqrt{\gamma}}\right) \\
& \sim+\frac{\lambda}{4} \tilde{\mathcal{K}}^{\mu \nu} K_{\mu \nu}+\frac{\lambda}{2}\left[\nabla^{\ell}\left(\tilde{\Delta}^{-1}\right)_{\ell}{ }^{k}\left(\epsilon_{i j k} \tilde{\mathcal{K}}^{i j}\right)\right]\left(\partial_{m} \tilde{\mathcal{K}}^{0 m}\right) .
\end{aligned}
$$

Substituting $\delta A_{0}$ given in (4.11) and using the GZ condition, one finds

$$
\begin{aligned}
\delta \mathcal{L} & =\partial_{0}\left[\frac{\lambda}{2} Z_{i} \epsilon^{i j k} \partial_{j} Z_{k}\right]+\frac{\lambda}{4} \tilde{\mathcal{K}}^{\mu \nu} K_{\mu \nu}+\delta_{\Phi} \mathcal{L}+\partial_{i} U^{i} \\
& =\partial_{0}\left[\frac{\lambda}{2} Z_{i} \epsilon^{i j k} \partial_{j} Z_{k}-\frac{\lambda}{2}\left(A_{i} \epsilon^{i j k} \partial_{j} A_{k}\right)\right]+\partial_{i} U^{i} \\
& =\partial_{\mu} U^{\mu}
\end{aligned}
$$

for some $U^{i}$. This establishes that the generic action becomes pseudo-invariant under the extended A-transformation in curved spaces. 
We have considered above the duality symmetry in the Lagrangian formalism. It is known that in the canonical formalism the symmetry can be realized simply as an invariance of the Hamiltonian. We argue here that the A-transformation can be described as a canonical transformation. Let $\left(A_{\mu}, \pi^{\mu}\right)$ be canonically conjugate variables, which are relevant to the duality transformation. From (4.16) the generator of the canonical transformation is given by

$$
\mathcal{W}=\frac{\lambda}{2} \int d^{3} x \sqrt{\gamma}\left[\frac{\pi^{i}}{\sqrt{\gamma}} D_{i \ell}^{-1} \frac{\pi^{\ell}}{\sqrt{\gamma}}+A_{i} D^{i j} A_{j}+\pi^{0} \delta A_{0}\right],
$$

with the identification, $\tilde{\mathcal{K}}^{0 i}=\pi^{i}$. It generates the transformations of the canonical variables

$$
\begin{aligned}
\delta A_{i} & =\lambda D_{i j}^{-1} \frac{\pi^{j}}{\sqrt{\gamma}} \\
\delta \pi^{i} & =-\lambda \epsilon^{i j k} \partial_{j} A_{k},
\end{aligned}
$$

which lead to the desired relations

$$
\begin{aligned}
\delta B^{i} & =\lambda \pi_{\perp}^{i}, \\
\delta \pi_{\perp}^{i} & =-\lambda B^{i},
\end{aligned}
$$

as well as the invariance of the Gauss law constraint, $\delta\left(\partial_{i} \pi^{i}\right)=0$. Note that the last term proportional to $\delta A_{0}$ in (4.17) contains "velocity variables" $\dot{q}$ in addition to phase space variables $(q, p)$. Such a term is interpreted as a generalized canonical quantity considered in ref.[9]. This term may induce new contributions to the canonical transformation of $\pi^{i}$ in (4.17). However since all of them are proportional to the constraint $\pi^{0} \approx 0$, they vanish on the constraint surface, and therefore have not been included there.

\section{Summary and Discussion}

In this paper we have shown that electric-magnetic duality can be formulated as a symmetry of actions. It is based on an integrated form of the duality transformation for the gauge field. Our reinterpretation of the GZ condition as the general criterion for the invariance of the action reveals its importance as a guiding principle in constructing selfdual actions for theories of interacting U(1) field strength. The question that one needs to address is that how the GZ condition restricts the form of the self-dual actions for given matter field content. In the absence of matters, Gibbons and Rasheed [3] 8] showed that there are as many self-dual actions as functions of a single variable. A related question is how inclusion of supersymmetry eliminates such ambiguity.

Although we have considered $\mathrm{SO}(2)$ duality, extension to $\mathrm{U}(\mathrm{n})$ duality for $n$ interacting Maxwell fields will be straightforward. This duality associated with the compact group may be lifted to the maximal non-compact group $\operatorname{Sp}(2 \mathrm{n}, \mathrm{R})$, by introducing scalar fields which belong to the coset space $\operatorname{Sp}(2 \mathrm{n}, \mathrm{R}) / \mathrm{U}(\mathrm{n})[1][2]$. They give a non-linear realization of the $\operatorname{Sp}(2 n, R)$. 
It is natural to expect that if the action is pseudo-invariant under an infinitesimal transformation for certain continuous symmetry, it is so under a finite transformation for the symmetry, as long as the latter is obtained by an integration of the former. As for the duality symmetry, this is of course the case in the A-transformation, but not in the F-transformation: the pseudo-invariance under the F-transformation does not lead to that under a finite F-transformation. Even for the infinitesimal transformation, the action can be pseudo-invariant only when it is expressed in terms of the gauge field as can be observed in the following:

$$
\begin{aligned}
\delta S=\lambda G_{F} S & =2 \lambda \int d^{4} x \boldsymbol{E} \cdot \boldsymbol{B}, \quad S=\int d^{4} x \frac{1}{2}\left(\boldsymbol{E}^{2}-\boldsymbol{B}^{2}\right), \\
G_{F} & =\mathbf{B} \frac{\delta}{\delta \mathbf{E}}-\mathbf{E} \frac{\delta}{\delta \mathbf{B}} .
\end{aligned}
$$

A finite transformation generates terms,

$$
e^{\lambda G_{F}} S=S+2 \lambda \int d^{4} x \boldsymbol{E} \cdot \boldsymbol{B}+\lambda^{2} G_{F} \int d^{4} x \boldsymbol{E} \cdot \boldsymbol{B}+\cdots .
$$

The third term goes back to the Maxwell action if we perform $G_{F}$ operation, while we do not know how to do it once the integrant is expressed in terms of the gauge field. This observation would resolve a "paradox" that the Maxwell action is not pseudo-invariant under a finite duality transformation, while it is so under an infinitesimal transformation. The source of the "paradox" is the use of the F-transformation. There is no such problem in the A-transformation.

An undesirable feature of our formulation is that locality as well as manifest $D=4$ covariance are lost. A way out of this drawback may be to introduce a new gauge field

whose curl gives the dual field strength $\tilde{K}$. Actually, the Schwarz-Sen model[5] and its covariant version of PST 6] retain the locality and the covariance in the duality rotation. In this approach, however, it is not clear if there exists the general criterion for selfdual actions such as the GZ condition. To find it, if exists, is certainly important for construction of manifestly self-dual actions in string and field theories.

\section{Acknowledgements}

K.I. would like to thank to the Yukawa Institute for its kind hospitality extended to him.

\section{A A derivation of the GZ condition as an algebraic relation}

In this appendix, we shall first derive (2.11) from the covariance of the EOM for matter fields (2.7), and then show that the GZ condition is an algebraic relation sensible even off the mass shell. 
Under the duality transformation (2.4) and (2.5), the EOM (2.7) transforms by

$$
\delta[\mathcal{L}]_{A}=\delta\left(\frac{\partial \mathcal{L}}{\partial \Phi^{A}}\right)-\partial_{\mu} \delta\left(\frac{\partial \mathcal{L}}{\partial \Phi_{\mu}^{A}}\right)
$$

where the first term on the r.h.s. reads

$$
\delta\left(\frac{\partial \mathcal{L}}{\partial \Phi^{A}}\right)=\delta \Phi^{B} \frac{\partial^{2} \mathcal{L}}{\partial \Phi^{B} \partial \Phi^{A}}+\delta \Phi_{\mu}^{B} \frac{\partial^{2} \mathcal{L}}{\partial \Phi_{\mu}^{B} \partial \Phi^{A}}+\frac{1}{2} \delta F \frac{\partial^{2} \mathcal{L}}{\partial F \partial \Phi^{A}} .
$$

Using (2.1) and (2.10), the last term in (A.2) is expressed as

$$
\begin{aligned}
\frac{1}{2} \delta F \frac{\partial^{2} \mathcal{L}}{\partial F \partial \Phi^{A}} & =\frac{\lambda}{4} \sqrt{-g} \frac{\partial}{\partial \Phi^{A}}(K \tilde{K}) \\
& =\sqrt{-g} \frac{\partial}{\partial \Phi^{A}}\left(\beta-\frac{\lambda}{4} F \tilde{F}-\delta \Phi^{B} \frac{\partial L}{\partial \Phi^{B}}-\delta \Phi_{\mu}^{B} \frac{\partial L}{\partial \Phi_{\mu}^{B}}\right) .
\end{aligned}
$$

It follows from (A.2) and (A.3) that

$$
\delta\left(\frac{\partial \mathcal{L}}{\partial \Phi^{A}}\right)=\sqrt{-g}\left(\frac{\partial \beta}{\partial \Phi^{A}}-\frac{\partial \xi^{B}}{\partial \Phi^{A}} \frac{\partial L}{\partial \Phi^{B}}-\Phi_{\mu}^{C} \frac{\partial^{2} \xi^{B}}{\partial \Phi^{C} \partial \Phi^{A}} \frac{\partial L}{\partial \Phi_{\mu}^{B}}\right) .
$$

Likewise, one finds

$$
\delta\left(\frac{\partial \mathcal{L}}{\partial \Phi_{\mu}^{A}}\right)=\sqrt{-g}\left(\frac{\partial \beta}{\partial \Phi_{\mu}^{A}}-\frac{\partial \xi^{B}}{\partial \Phi^{A}} \frac{\partial L}{\partial \Phi_{\mu}^{B}}\right) .
$$

Then, one obtains (2.11) from (A.1), (A.4) and (A.5).

Although the GZ condition has been obtained originally via the F-transformation, it is an algebraic relation that is free from the on-shell conditions. To see this explicitly, we consider off-shell extended transformations inferred by the A-transformation:

$$
\hat{\delta} F=\lambda K+[E O M], \quad \hat{\delta} K=-\lambda F+[E O M]
$$

where $[E O M]$ denotes terms proportional to the EOM of the gauge fields. All such terms vanish on the mass shell, and we do not need their precise form below. Covariance relation of the EOM for matters may be replaced by

$$
\hat{\delta}[\mathcal{L}]_{A}=-\frac{\partial \xi^{B}}{\partial \Phi^{A}}[\mathcal{L}]_{B}+[E O M]
$$

One obtains then

$$
\begin{aligned}
\hat{\delta} \tilde{K}= & -\lambda \tilde{F}+\frac{\partial}{\partial F}\left\{\frac{\lambda}{4}(\tilde{K} K+\tilde{F} F)+\delta_{\Phi} L\right\}+\frac{1}{2} \frac{\partial \tilde{K}}{\partial F}[E O M] \\
\hat{\delta}[\mathcal{L}]_{A}= & -\frac{\partial \xi^{B}}{\partial \Phi^{A}}[\mathcal{L}]_{B}+\left[\sqrt{-g}\left\{\frac{\lambda}{4}(\tilde{K} K+\tilde{F} F)+\delta_{\Phi} L\right\}\right]_{A} \\
& +\frac{1}{2}[\sqrt{-g} \tilde{K}]_{A}[E O M] .
\end{aligned}
$$

Consistency of (A.8) with (A.6) and (A.7) leads to again the GZ condition:

$$
\frac{\lambda}{4}(F \tilde{F}+K \tilde{K})+\delta_{\Phi} L=0 .
$$




\section{B Basic formulae in $\mathrm{D}=3$ covariant calculations}

We illustrate the Laplacian operator $\tilde{\Delta}^{j}{ }_{i}$ acting on a vector by considering an integration of the curl

$$
\partial_{j} A_{k}-\partial_{k} A_{j}=\nabla_{j} A_{k}-\nabla_{k} A_{j}=\epsilon_{i j k} B^{i}
$$

where $\nabla_{i}$ is the covariant derivative with respect to $\mathrm{D}=3$ spatial metric $g_{i j}$ whose determinant is $\gamma$. The anti-symmetric tensor densities $\epsilon^{i j k}$ and $\epsilon_{i j k}$ are related with the $\mathrm{D}=4$ tensor densities by $\epsilon^{0 i j k}=\epsilon^{i j k}$ and $\epsilon_{i j k 0}=\epsilon_{i j k}$ with $\epsilon^{123}=\epsilon_{123}=1$. We also use the covariantly constant anti-symmetric tensors given by $\eta^{i j k}=\epsilon^{i j k} / \sqrt{\gamma}$, and $\eta_{i j k}=\epsilon_{i j k} \sqrt{\gamma}$. The Ricci tensor is defined by

$$
g^{i j}\left[\nabla_{i}, \nabla_{k}\right] A_{j}=R_{k}^{\ell} A_{\ell} .
$$

For $\mathrm{D}=3$ spaces, the curvature tensor is expressed in terms of the Ricci tensor by

$$
R_{i j k \ell}=\epsilon_{i j m} \epsilon_{k \ell n}(-\gamma)\left(R^{m n}-\frac{g^{m n}}{2} R\right)
$$

Using (B.2) and imposing the transverse condition,

$$
\nabla^{i} A_{i}=0
$$

one obtains

$$
\tilde{\Delta}_{i}^{j} A_{j}=\nabla^{k} \epsilon_{k i \ell} B^{\ell}
$$

Assuming the presence of the inverse operator, it is solved as

$$
A_{i}=\left(\tilde{\Delta}^{-1}\right)_{i}^{j} \nabla^{k} \epsilon_{k j \ell} B^{\ell}
$$

For a vector $T_{i}$, we have

$$
\begin{aligned}
\nabla^{\ell}\left(\tilde{\Delta}^{-1}\right)_{\ell}{ }^{k} T_{k} & =\left(\Delta^{-1}\right) \nabla^{k} T_{k}, \\
\eta^{i j k} \nabla_{j}\left(\tilde{\Delta}^{-1}\right)_{k}^{\ell} T_{\ell} & =\left(\tilde{\Delta}^{-1}\right)^{i}{ }_{j} \eta^{j k \ell} \nabla_{k} T_{\ell},
\end{aligned}
$$

where the curvature relation $(\mathbb{B . 3})$ is used to derive the last equation. The tensor operator $D$ and its inverse $D^{-1}$ defined in the projected space by

$$
\begin{aligned}
D^{i j} & =\eta^{i k j} \nabla_{k}=\nabla_{k} \eta^{i k j} \\
D_{i \ell}^{-1} & =\left(\tilde{\Delta}^{-1}\right)_{i}{ }^{k} \nabla^{j} \eta_{j k \ell}=\eta_{i j k} \nabla^{k}\left(\tilde{\Delta}^{-1}\right)_{\ell}^{j}
\end{aligned}
$$

which satisfy

$$
\begin{aligned}
D_{i m}^{-1} D^{m k} T_{k} & =\left[\left(\tilde{\Delta}^{-1}\right)_{i}{ }^{k} \nabla^{\ell}-\left(\tilde{\Delta}^{-1}\right)_{i}{ }^{\ell} \nabla^{k}\right] \nabla_{\ell} T_{k} \\
& =\left[\delta_{i}{ }^{k}-\left(\tilde{\Delta}^{-1}\right)_{i}{ }^{\ell} \nabla_{\ell} \nabla^{k}\right] T_{k} \equiv O_{i}{ }^{k}(\nabla) T_{k},
\end{aligned}
$$


and

$$
\begin{aligned}
\nabla^{k} D_{k \ell} T^{\ell} & =\nabla^{k}\left(\tilde{\Delta}^{-1}\right)_{k}^{r} \nabla^{s} \epsilon_{s r \ell} \sqrt{\gamma} T^{\ell} \\
& =\left(\Delta^{-1}\right) \frac{\epsilon^{s r \ell}}{\sqrt{\gamma}}\left(-R_{\ell r s}^{m}\right) T_{m}=0
\end{aligned}
$$

where we have used the Bianchi identity in the last line in $(\mathbb{B} .10)$. The partial integration formula for the inverse Laplacian operator that the tensor operators read

$$
\begin{aligned}
\int d^{3} x \sqrt{\gamma}\left[T^{\ell}\left(\tilde{\Delta}^{-1}\right)_{\ell}^{k} S_{k}\right] & \left.=\int d^{3} x \sqrt{\gamma}\left[\left(\left(\tilde{\Delta}^{-1}\right)^{k} T^{\ell}\right) S_{k}\right)\right] \\
\int d^{3} x \sqrt{\gamma} S^{k} D_{k \ell}^{-1} T^{\ell} & =\int d^{3} x \sqrt{\gamma} T^{k} D_{k \ell}^{-1} S^{\ell} \\
\int d^{3} x \sqrt{\gamma} S_{k} D^{k \ell} T_{\ell} & =\int d^{3} x \sqrt{\gamma} T_{k} D^{k \ell} S_{\ell} .
\end{aligned}
$$

The second equation is derived, for instance, as

$$
\begin{aligned}
\int d^{3} x \sqrt{\gamma}\left[T^{k} D_{k \ell}^{-1} S^{\ell}\right] & =-\int d^{3} x \sqrt{\gamma}\left[\left\{\nabla^{j}\left(\tilde{\Delta}^{-1}\right)_{\ell}^{k} T^{\ell}\right\} \epsilon_{j k n} \sqrt{\gamma} S^{n}\right] \\
& =-\int d^{3} x\left[\left(\tilde{\Delta}^{-1}\right)_{m}^{k}\left(\sqrt{\gamma} \epsilon_{k \ell r}\right) \nabla^{\ell} T^{r}\right]\left(\sqrt{\gamma} S^{m}\right) \\
& =+\int d^{3} x \sqrt{\gamma}\left[S^{\ell} D_{\ell n}^{-1} T^{n}\right] .
\end{aligned}
$$

\section{Proof of the on-shell relation in curved spaces}

In this Appendix, we show that the relation

$$
\delta F_{0 i}=\lambda K_{0 i}+\lambda D_{i j}^{-1}\left(\frac{\partial_{\mu} \tilde{\mathcal{K}}^{\mu j}}{\sqrt{\gamma}}\right)+\lambda O_{i}{ }^{k} \partial_{0}\left(\frac{D_{k j}^{-1}}{\sqrt{\gamma}}\right) \tilde{\mathcal{K}}^{0 j}
$$

reduces to $\delta F_{0 i}=\lambda K_{0 i}$ on the mass shell. The second term on the r.h.s. of (C.1) is proportional to EOM (2.3). One rewrites the third term by using the projection property $\delta_{i}^{k}=D_{i m}^{-1} D^{m k}+\nabla_{i}\left(\Delta^{-1}\right) \nabla^{k}$ :

$$
\partial_{0}\left(\frac{D_{i j}^{-1}}{\sqrt{\gamma}}\right) \tilde{\mathcal{K}}^{0 j}=\partial_{0}\left(\frac{D_{i j}^{-1}}{\sqrt{\gamma}}\right) \quad\left(D^{j k} \sqrt{\gamma} \frac{D_{k \ell}^{-1}}{\sqrt{\gamma}}+\nabla^{j} \frac{1}{\Delta} \nabla_{\ell}\right) \tilde{\mathcal{K}}^{0 \ell},
$$

where the last term is proportional the Gauss law. Define

$$
Y_{i} \equiv D_{i j}^{-1} \frac{\tilde{\mathcal{K}}^{0 j}}{\sqrt{\gamma}}
$$

and note that $\sqrt{\gamma} D^{j k}$ has no time dependence when it operates on $Y_{k}$ :

$$
\sqrt{\gamma} D^{j k} Y_{k}=\epsilon^{j i k} \nabla_{i} Y_{k}=\epsilon^{j i k} \partial_{j} Y_{k}
$$


Then, it follows that

$$
\begin{aligned}
\partial_{0}\left(\frac{D_{i j}^{-1}}{\sqrt{\gamma}}\right) D^{j k} \sqrt{\gamma} Y_{k} & =\partial_{0}\left(\delta_{i}^{k}-\nabla_{i} \frac{1}{\Delta} \nabla^{k}\right) Y_{k} \\
& =-\partial_{0}\left(\nabla_{i} \frac{1}{\Delta} \nabla^{k}\right) Y_{k}=\nabla_{i} \frac{1}{\Delta} \partial_{0}\left(\nabla^{k}\right) Y_{k},
\end{aligned}
$$

where we have used the transverse condition, $\nabla^{k} Y_{k}=0$. The third term in (C.1) becomes then

$$
\begin{aligned}
O_{i}{ }^{k} \partial_{0}\left(D_{k j}^{-1} \frac{1}{\sqrt{\gamma}}\right) \tilde{\mathcal{K}}^{0 j}= & O_{i}{ }^{j}\left[-\nabla_{j} \frac{1}{\Delta} \partial_{t}\left(\nabla^{k}\right)\left(D_{k \ell}^{-1} \frac{\tilde{\mathcal{K}}^{0 \ell}}{\sqrt{\gamma}}\right)\right] \\
& +O_{i}{ }^{j}\left[\partial_{0}\left(\frac{D_{j m}^{-1}}{\sqrt{\gamma}}\right) \nabla^{m} \frac{1}{\Delta}\left(\partial_{\ell} \tilde{\mathcal{K}}^{0 \ell}\right)\right] .
\end{aligned}
$$

Here the first vanishes because of the projection property of $O_{i}{ }^{j}$, and the second term is proportional to the Gauss law. One then arrives at the on-shell relation, $\delta F_{0 i}=\lambda K_{0 i}$.

\section{References}

[1] M.K.Gaillard and B.Zumino, Duality Rotations for Interacting Fields, Nucl. Phys.B193 (1981) 221-244.

[2] M.K.Gaillard and B.Zumino, Self-Duality in Nonlinear Electromagnetism, hepth/9705226; Nonlinear Electromagnetic Self-Duality and Legendre Transformations, hep-th/9712103.

[3] G. W. Gibbons and D. A. Rasheed, Electric-magnetic duality rotations in non-linear electrodynamics, Nucl. Phys. B454 (1995) 185-206.

[4] S.Deser and C.Teitelboim, Duality transformations of Abelian and non-Abelian gauge fields, Phys. Rev. D13 (1976) 1592-1597.

[5] J.H.Schwarz and A.Sen, Duality symmetric actions, Nucl. Phys.B411 (1994) 35-63.

[6] P.Pasti, D.Sorokin and M.Tonin, Duality symmetric actions with manifest space-time symmetries, Phys. Rev. D52 (1995) R4277-4281.

[7] Y.Igarashi, K.Itoh and K.Kamimura, Self-Duality in Super D3-Brane Action, YITP98-24, TOHO-FP-9860

[8] G. W. Gibbons and D. A. Rasheed, SL(2,R) invariance of non-linear electrodynamics coupled to an axion and a dilaton, Phys. Lett. B365 (1996) 46-50.

[9] K.Kamimura Singular Lagrangian and Constrained Hamiltonian Systems, Generalized Canonical Formalism, Nuovo Cimento 68B (1982) 33-54.

[10] I.Bengtsson, Manifestly Duality in Born-Infeld Theory, hep-th/9612174;

D.Berman, SL(2,Z) duality of Born-Infeld theory from non-linear self-dual electrodynamics in 6 dimensions, hep-th/9706208;

A.Khoudier and Y. Parra, On Duality in the Born-Infeld Theory, hep-th/9708011. 